\title{
Variations in the magnetic properties of meteoritic cloudy zone
}

Claire I. O. Nichols*, James F. J. Bryson, Roberts Blukis, Julia Herrero-Albillos, Florian

Kronast, Rudolf Rüffer, Aleksandr I. Chumakov, Richard J. Harrison

*Corresponding author: cion2@mit.edu

This article has been accepted for publication and undergone full peer review but has not been through the copyediting, typesetting, pagination and proofreading process, which may lead to differences between this version and the Version of Record. Please cite this article as doi: 10.1029/2019GC008798

(C2020 American Geophysical Union. All rights reserved. 


\section{meteoritic cloudy zone}

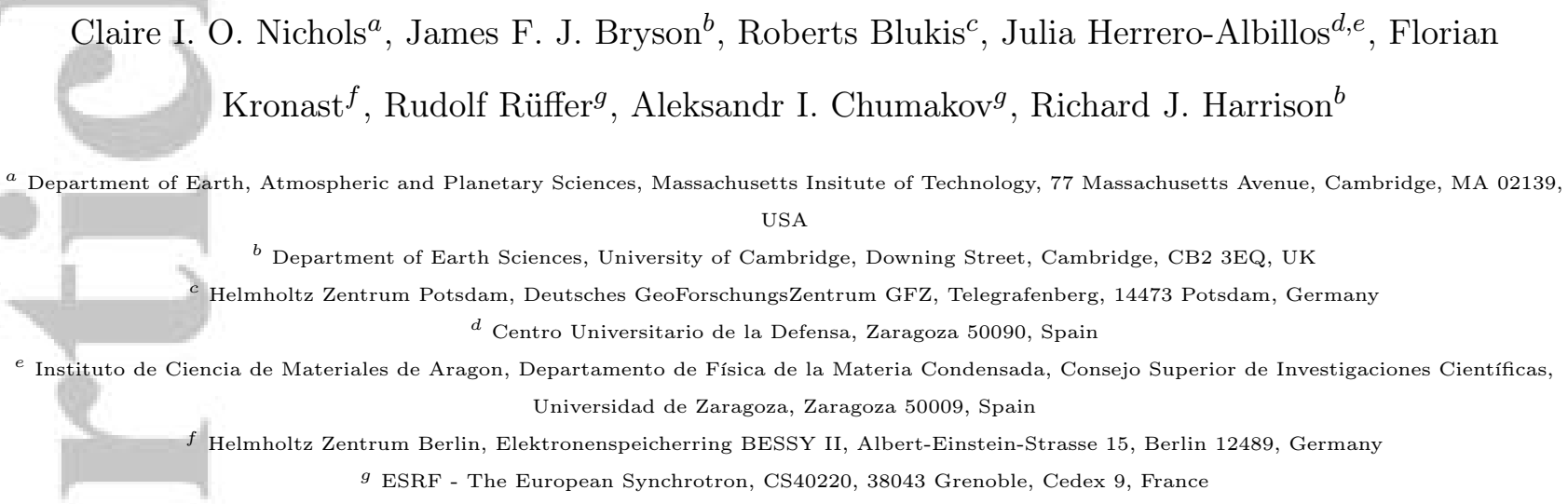

Key Words: Meteorites, Rock magnetism, Paleomagnetism

Key Point: Meteoritic cloudy zone formed at intermediate cooling rates can acquire a stable and reliable nanopaleomangetic remanence.

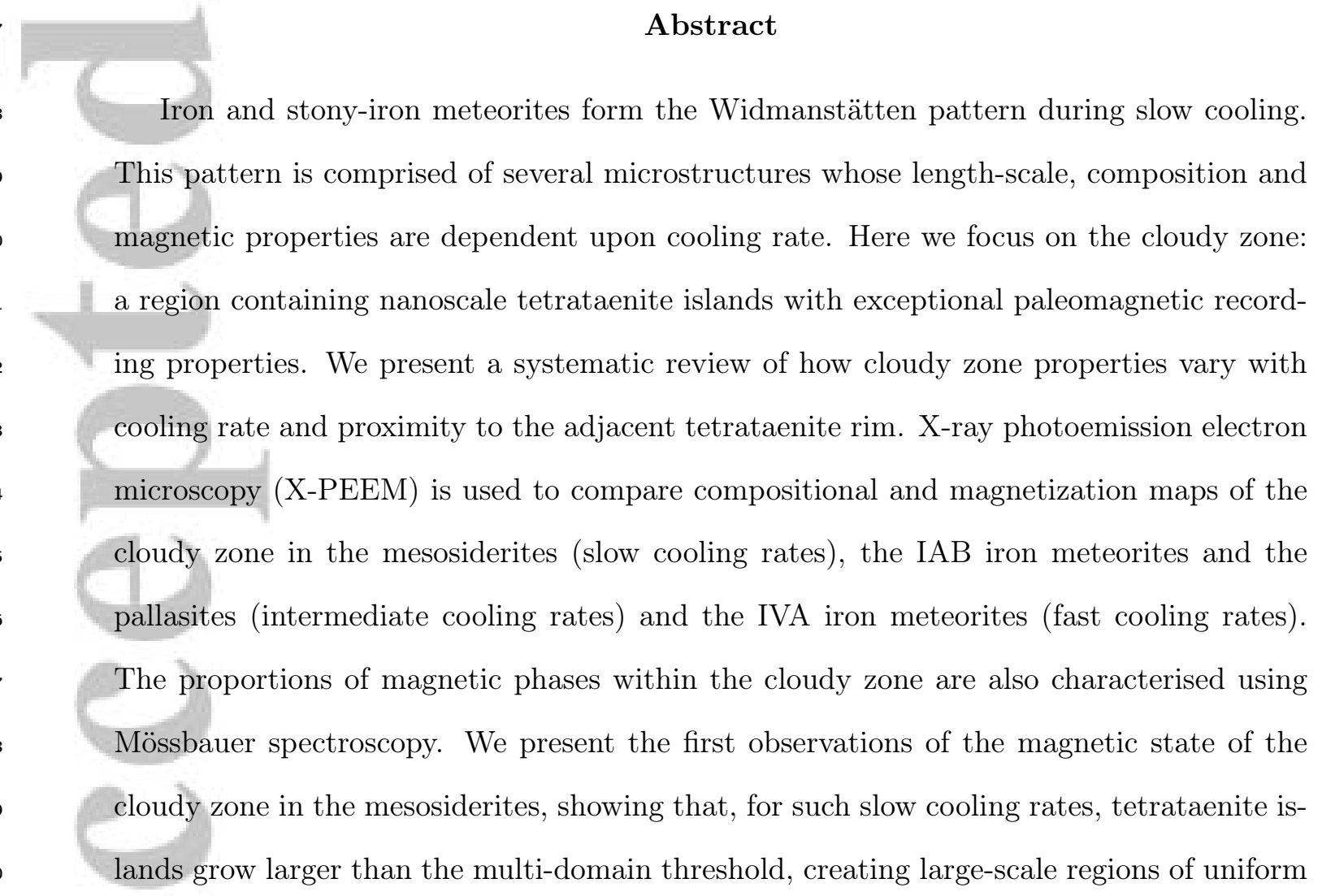

Iron and stony-iron meteorites form the Widmanstätten pattern during slow cooling. This pattern is comprised of several microstructures whose length-scale, composition and magnetic properties are dependent upon cooling rate. Here we focus on the cloudy zone: a region containing nanoscale tetrataenite islands with exceptional paleomagnetic recording properties. We present a systematic review of how cloudy zone properties vary with cooling rate and proximity to the adjacent tetrataenite rim. X-ray photoemission electron microscopy (X-PEEM) is used to compare compositional and magnetization maps of the cloudy zone in the mesosiderites (slow cooling rates), the IAB iron meteorites and the pallasites (intermediate cooling rates) and the IVA iron meteorites (fast cooling rates). The proportions of magnetic phases within the cloudy zone are also characterised using Mössbauer spectroscopy. We present the first observations of the magnetic state of the cloudy zone in the mesosiderites, showing that, for such slow cooling rates, tetrataenite islands grow larger than the multi-domain threshold, creating large-scale regions of uniform 
magnetization across the cloudy zone that render it unsuitable for paleomagnetic analysis. For the most rapidly cooled IVA meteorites, the time available for Fe-Ni ordering is insufficient to allow tetrataenite formation, again leading to behaviour that is unsuitable for paleomagnetic analysis. The most reliable paleomagnetic remanence is recorded by meteorites with intermediate cooling rates $\left(\sim 2-500^{\circ} \mathrm{C} \mathrm{Myr}^{-1}\right)$ which produces islands that are 'just right' both in size and degree of Fe-Ni order.

\section{Introduction}

Iron meteorites are characterised by the highly distinctive Widmanstätten pattern, a complex growth of Fe-Ni microstructures revealed by polishing and etching the metallic surface. The Widmanstätten pattern forms during slow cooling; the full range of microstructures are observed for cooling rates below $\sim 10,000{ }^{\circ} \mathrm{C} \mathrm{Myr}^{-1}$ (Yang et al., 1997, 2010). Kamacite $(\alpha)$, the body centered cubic (bcc) Fe-rich endmember, nucleates and begins to grow upon cooling through $\sim 900{ }^{\circ} \mathrm{C}$. This phase makes up the bulk of the meteoritic metal and its large grain size means it is magnetically multidomain. The nucleation of kamacite causes Ni to be partitioned into the surrounding face centered cubic (fcc) taenite $(\gamma)$. An 'M'-shaped Ni-diffusion profile develops, with highest $\mathrm{Ni}$ concentrations of $\sim 50 \mathrm{wt} \%$ immediately adjacent to the kamacite. As Ni content drops below $\sim 47 \mathrm{wt} \%$, spinodal decomposition takes place forming a mixture of nanoscale $\mathrm{Fe}_{0.5} \mathrm{Ni}_{0.5}$ islands in an Fe-rich matrix. This microstructure is known as the cloudy zone. As $\mathrm{Ni}$ content continues to decrease to $\sim 25 \mathrm{wt} \%$, spinodal decomposition is initiated at lower temperatures. Islands are largest $(<\sim 500 \mathrm{~nm})$ in the most Ni-rich regions (Yang et al., 1997; Maurel et al., 2019). Regions with $<25$ wt\% Ni, below the stability of the spinodal region, undergo a martensitic transition, spontaneously transforming from fcc taenite to bcc martensite. The temperature of the martensitic transition increases with decreasing Ni content. If the transition occurs at a high temperature and the cooling rate is slow, the martensite has enough time upon further cooling to exsolve into plessite, a complex intergrowth of kamacite and taenite (Goldstein and Michael, 2006).

The cloudy zone has been shown to be an excellent paleomagnetic recorder (Uehara et al., 
2011; Bryson et al., 2014b; Einsle et al., 2018). As the islands cool below $320^{\circ} \mathrm{C}$ they transform to ordered tetrataenite. Ordering occurs parallel to the $\{100\}$ planes and the magnetic easy axis is aligned perpendicular to the ordered planes. The cloudy zone islands acquire a chemical transformation remanent magnetization (CTRM) which is extremely stable. Thermal overprints at relatively low temperatures $\left(>\sim 360{ }^{\circ} \mathrm{C}\right)$ are easily observable since they can dissolve the cloudy zone entirely (Goldstein et al., 2009b), and the high intrinsic coercivity of the cloudy zone (>1 T; Uehara et al., 2011) means an isothermal remanent magnetization (IRM) overprint is unlikely since naturally-occurring magnetic fields are rarely this strong.

The size of the islands in the cloudy zone depends on the cooling rate; the faster the cooling, the smaller the islands and this relationship is empirically well defined by the equation $d_{C Z}^{2.9}=\frac{k}{C R}$ where $d_{C Z}$ is the diameter of the largest cloudy zone islands in $\mathrm{nm}, k$ is a constant, equal to $7,620,000{ }^{\circ} \mathrm{C} \mathrm{nm}{ }^{2.9} \mathrm{Myr}^{-1}$ and $C R$ is cooling rate in ${ }^{\circ} \mathrm{C} \mathrm{Myr}^{-1}$ for a temperature range of $500-700{ }^{\circ} \mathrm{C}$ (Yang et al., 2010). Cooling rates are determined by measuring the diameter of tetrataenite islands within the cloudy zone and the width of the tetrataenite rim in etched samples (Yang et al., 1997, 2008, 2010; Goldstein et al., 2009a). More accurate cooling rate estimates are obtained by measuring the shape of the 'M'-shaped Ni diffusion profile across kamacite-taenite interfaces using electron probe microanalysis (EPMA) (Goldstein et al., 2014). Island size also decreases with distance from the tetrataenite rim. Since islands form by spinodal decomposition, as opposed to nucleation and growth, islands spontaneously form at $\leq 85 \%$ of their present day size (Maurel et al., 2019). The first islands to form are closest to the tetrataenite rim where the Ni content is highest. The Ni content of the cloudy zone affects the temperature at which it enters the spinodal region; at lower Ni contents - and therefore lower spinodal initiation temperatures — smaller islands are formed.

The cooling rate during cloudy zone formation controls the ability of this microstructure to record paleomagnetic information. Cooling rate affects island size, the degree of ordering (tetrataenite vs taenite) and packing fraction, which in turn controls the strength of interactions between islands. In this paper we will describe the optimal cloudy zone for recording paleomagnetic information, dictated by both cooling rate and distance from the tetrataenite 


\begin{tabular}{llccl}
\hline Meteorite & Type & TT Islands (nm) & $\mathbf{C R}\left({ }^{\circ} \mathbf{C ~} \mathbf{M y r}^{-1}\right)$ & References \\
\hline \hline Bishop Canyon & IVA Iron & 12 & $2500 \pm 1.3$ & Yang et al. (2007) \\
Steinbach & IVA Iron & 29 & 150 & Goldstein et al. (2009b) \\
Chinautla & IVA Iron & 32 & $110 \pm 1.7$ & Goldstein et al. (2009b) \\
Tazewell & IAB Iron & $90-100$ & 20.8 & Goldstein et al. (2014) \\
Toluca & IAB Iron & $100-120$ & 11.6 & Goldstein et al. (2014) \\
Marjalahti & MG Pallasite & $118 \pm 3$ & $7.6 \pm 0.6$ & Yang et al. (2010) \\
Odessa & IAB Iron & $120-300$ & 11.6 & Nichols et al. (2018) \\
Brenham & MG Pallasite & $123 \pm 3$ & $6.2 \pm 0.9$ & Yang et al. (2010) \\
Springwater & MG Pallasite & $132 \pm 3$ & $5.4 \pm 0.5$ & Yang et al. (2010) \\
Imilac & MG Pallasite & $143 \pm 4$ & $4.3 \pm 0.3$ & Yang et al. (2010) \\
Esquel & MG Pallasite & $157 \pm 11$ & $3.3 \pm 0.6$ & Yang et al. (2010) \\
Estherville & Mesosiderite & $463 \pm 32$ & $0.2-0.5$ & Goldstein et al. (2014) \\
\hline
\end{tabular}

Table 1: Table summarizing the meteorites reviewed in this study including the largest tetrataenite island diameters (TT Islands) and their cooling rates at $\sim 500{ }^{\circ} \mathrm{C}(\mathrm{CR})$. 'MG pallasite' refers to pallasite meteorites from the Main Group. 


\section{Sample Characterisation}

\subsection{The Mesosiderites}

The mesosiderites are an unusual group of stony-iron meteorites, comprised of FeNi metal and a range of brecciated basaltic, gabbroic, dunitic and orthopyroxene-rich clasts (Greenwood et al., 2015). The composition of the silicates, combined with FeNi metal, suggests the mesosiderites represent a mixture of core and crustal material. The oxygen isotope composition of the silicates has been used to infer their origin on the same parent body, and their well-mixed compositions and brecciated nature imply they represent a regolith breccia (Mittlefehldt, 1980). It has been proposed that an impact event mixed the molten core of an impactor with the regolith breccia, forming the mesosiderites.

An unresolved issue is the exceptionally slow cooling rates of the mesosiderites, which is slower than that of any other meteorite group. Initially, the mesosiderites cooled rapidly; Fe$\mathrm{Mg}$ profiles in pyroxenes suggest cooling rates of $1-100{ }^{\circ} \mathrm{C} \mathrm{yr}^{-1}$ between $1150-900{ }^{\circ} \mathrm{C}$ and plagioclase overgrowth textures predict a cooling rate $\geq 0.1{ }^{\circ} \mathrm{C} \mathrm{yr}^{-1}$ between $1100-850{ }^{\circ} \mathrm{C}$ (Delaney et al., 1980; Ruzicka et al., 1994). This initial fast cooling rate is attributed to rapid thermal equilibration between hot and cold ejecta during the impact event (Scott et al., 2001). At temperatures below $400{ }^{\circ} \mathrm{C}$, cooling rates drop to $0.2-0.5{ }^{\circ} \mathrm{C} \mathrm{Myr}^{-1}$ based on $\mathrm{Ni}$ and $\mathrm{Co}$ concentrations at kamacite-taenite interfaces (Goldstein et al., 2014; Wasson and Hoppe, 2014). This is supported by the large size of the cloudy zone microstructure - tetrataenite islands are $400-450 \mathrm{~nm}$ in diameter (Goldstein et al., 2009a) — yielding a cooling rate estimate of $0.5{ }^{\circ} \mathrm{C} \mathrm{Myr}^{-1}$ (Yang et al., 1997). Fe-Mg ordering in orthopyroxene and ${ }^{40} \mathrm{Ar} /{ }^{39} \mathrm{Ar}$ ages also support very slow cooling (Ganguly et al., 1994; Bogard and Garrison, 1998). These extremely slow cooling rates are difficult to reconcile with the inference that the metal mixed with crustal and regolith material near the parent body surface. It has been proposed that after their formation, the mesosiderites were buried deep inside their parent body, which must be well-insulated by a thick regolith layer (Scott et al., 2001; Greenwood et al., 2015). Samples BM 65575 and BM 53764 of the Estherville mesosiderite were borrowed from the Natural 
History Museum, London.

\section{$2.2 \quad$ The Pallasites}

The pallasites consist of an intimate mix of FeNi metal and large (cm-sized) olivine crystals and were initially considered to originate from the core-mantle boundary of a differentiated asteroid. It has since been shown that their variable cooling rates and paleomagnetic record of an internal core dynamo firmly rule out an origin deep within a planetesimal interior (Yang et al., 2010; Tarduno et al., 2012). It is now proposed that the pallasites were formed by an impact between two differentiated planetesimals, resulting in the molten core of one being injected into the mantle of the other. The significant difference in density between silicate and metal suggests that the two phases should quickly separate; their intimate mix therefore suggests initial rapid cooling following the intrusion of molten metal. Further impact events and the addition of a regolith layer to the parent body could explain late, slow cooling below $700{ }^{\circ} \mathrm{C}$ of $2.5-18^{\circ} \mathrm{C} \mathrm{Myr}^{-1}$ (Yang et al., 2010).

Samples of Brenham (BM 68725) and Marjalahti (BM 1920,318) were obtained from the Natural History Museum, London. Previous magnetic and compositional characterisation of these samples are reported by Nichols et al. (2016). Paleomagnetic results revealed evidence for cessation of a dynamo on the pallasite parent body after a thermally convective dynamo and prior to a compositional dynamo driven by core solidification.

\subsection{The IAB Iron Meteorites}

The IAB iron meteorites are an unusual group, since they are not thought to form via fractional crystallization in a planetary core (Benedix et al., 2014). They contain silicate fragments with both primitive achondritic and chondritic compositions, which supports the hypothesis that the IAB parent body was partially differentiated (Benedix et al., 2000). It is likely that the IAB irons formed as isolated pools of metal after an impact event disrupted the process of planetary differentiation (Schulz et al., 2012). The IAB iron meteorites are rich in carbon, and exhibit unusual FeNi microstructures (Buchwald, 1975; Goldstein et al., 2017; Nichols et al., 2018). 
Samples of Toluca (TN 4389), Odessa (11538) and Tazewell (16269) were borrowed from the Sedgwick Museum, University of Cambridge. These samples have previously been studied using X-PEEM and results are reported in Nichols et al. (2018) and Bryson et al. (2014b). Paleomagnetic results reported by Nichols et al. (2018) suggested the IAB parent body did not fully differentiate and did not have a substantial metallic core capable of generating a dynamo.

\subsection{The IVA Iron Meteorites}

The IVA iron meteorites are thought to originate from a metallic core that had its overlying silicate mantle removed by glancing collisions during the early solar system. Without the insulation provided by a silicate mantle, the parent core of the IVAs is expected to have cooled far quicker than most other iron meteorites. Indeed, the sizes of the low-temperature microstructures within these meteorites are the smallest that have been measured, with cooling rates ranging from $100-10,000{ }^{\circ} \mathrm{C} \mathrm{Myr}^{-1}$ (Yang et al., 2008). Furthermore, unlike Earth's core, the rapid cooling rate of the surface of the IVA parent core is expected to result in initial solidification at the asteroid surface, and the crystallisation front will then have advanced toward the centre of the core as cooling continued. The solidification of this core therefore represents a fundamentally different regime to that of our own planet and could provide crucial constraints on dynamo generation (Neufeld et al., 2019).

A sample of the Steinbach IVA iron meteorite, sample number BM 35540 was acquired from the Natural History Museum, London. Previous X-PEEM imaging and bulk paleomagnetic measurements on this sample are reported by Bryson et al. (2017). Paleomagnetic results revealed the first direct evidence of inward core solidification within an asteroid.

\section{Methods}

\subsection{Sample Preparation}

Samples were prepared in the Department of Earth Sciences, University of Cambridge, by cutting $\sim 5 \times 5 \mathrm{~mm}$ sections using a tile-cutting saw under running water to keep the samples 
cool. Samples were then ground down until $<1 \mathrm{~mm}$ thick and polished, down to $0.25 \mu \mathrm{m}$-grade diamond paste. They were subsequently etched for $\sim 20$ seconds using nital (2\% nitric acid in ethanol) and examined using a reflected light microscope to check for signs of alteration or shock. Samples were repolished to reveal a fresh surface prior to X-PEEM measurement. They were then sputtered for $\sim 18$ hours using a focussed Ar-ion beam under ultra-high vacuum (pressure $<1.5 \times 10^{-5}$ mbar) while gradually decreasing the voltage from $1.2 \mathrm{keV}$ to $0.4 \mathrm{keV}$ to remove any oxidation or surface magnetization induced by polishing. Samples were kept in vacuum between sputtering and measuring (measuring pressure $<1.0 \times 10^{-8}$ mbar).

\subsection{X-ray Photoemission Electron Microscopy (X-PEEM)}

X-PEEM was used to collect images with nanoscale resolution of the composition and magnetization of iron meteorites. X-PEEM was performed at the SPEEM UE49 beamline, BESSY II (Berlin, Germany). An intense beam of monochromatic X-rays is focussed at an angle of $16^{\circ}$ to the sample surface. Secondary photoelectrons are excited by the X-rays from the top $\sim 5 \mathrm{~nm}$ of the sample surface. This technique allows compositional and magnetic imaging to be carried out in the same location in quick succession by changing the energy and polarisation of the X-ray beam, meaning a direct comparison can be made between composition and magnetization for each region. Each image is individually assessed for light drift and charging artefacts both qualitatively and quantitatively by comparing light intensity in sequential images using a Student T-test; only the highest quality data are selected for further analysis. 160 images (80 for each polarity, or change in energy) are acquired with a 2 second exposure time per image. These images are then aligned by selecting a surface feature as a reference point, and averaged to improve signal to noise ratio in the final images.

Compositional images were acquired using linearly polarised X-rays tuned to the energy of the $\mathrm{Fe}_{3}$ and $\mathrm{Ni} \mathrm{L}_{3}$ edges ( $~ 707 \mathrm{eV}$ and $\sim 852 \mathrm{eV}$, respectively). The energy of the X-ray beam is fine-tuned by imaging the sample over a range of energies and precisely locating the $\mathrm{L}_{3}$ peaks.

The $\mathrm{L}_{3}$ peaks are used since they provide maximum compositional contrast when conducting $\mathrm{X}$-PEEM. Images were also acquired at pre-edge energies $(\sim 700 \mathrm{eV}$ and $\sim 845 \mathrm{eV}$ for Fe and 
$\mathrm{Ni}$, respectively). Each on-edge image was normalised by dividing by its equivalent pre-edge image. The Ni image was divided by the Fe image in order to generate a semi-quantitative map of $\mathrm{Ni} / \mathrm{Fe}$ ratio.

\subsubsection{X-ray Magnetic Circular Dichroism (XMCD)}

XMCD was used in combination with X-PEEM to image the magnetization state at the energy of the $\mathrm{Fe} \mathrm{L}_{3}$ edge (Stöhr et al., 1998; Ohldag et al., 2001). XMCD intensity is a projection of the magnetization onto the incident direction of the X-ray beam; negative intensities represent magnetization towards the X-ray beam and are coloured red, while positive intensities (magnetization parallel to the beam) are blue. magnetization perpendicular to the beam, as well as non-magnetic material appears white. Images were acquired for both left and right circularly polarised X-rays, which excite electrons in opposite spin states. Since spin state directly correlates to the direction of magnetization, this generates contrast depending on the component of magnetization parallel to the X-ray beam. Images were taken along parallel sections of the tetrataenite rim in order to be directly comparable. Images taken in each polarisation state were subtracted from one another and divided by their sum, in order to normalise and enhance magnetic contrast. Thus,

$$
I=\frac{I_{R}-I_{L}}{I_{R}+I_{L}}
$$

where $I_{R}$ and $I_{L}$ are right and left polarised X-ray intensities, respectively. $I$ is the resulting XMCD intensity (Bryson et al., 2014b).

\subsection{Mössbauer spectroscopy}

Samples of Tazewell, Esquel and Estherville were prepared by mechanical polishing on a carbide paper to the desired thickness of $20-40 \mu \mathrm{m}$. Samples were further mechanically polished with water based monocrystalline diamond suspension, with the final particle diameter of $0.25 \mu \mathrm{m}$. Synchrotron Mössbauer spectroscopy was performed with the Synchrotron Mössbauer Source (SMS) (Smirnov et al., 1997; Potapkin et al., 2012) at the Nuclear Resonance beamline ID18 
(Rüffer and Chumakov, 1996) at the European Synchrotron Radiation Facility in Grenoble, France. The beam from SMS is nearly $100 \%$ polarized with the electric vector in the vertical direction. Typical count rates on the sample reach $16 \mathrm{kHz}$ resonant quanta. The beam can be focused to a spot size of $9 \times 4 \mu \mathrm{m}^{2} \mathrm{FWHM}$ under ideal conditions (Rüffer and Chumakov, 1996). For our experiment, the beam shape is approximately Gaussian with a focused beam size $[\mathrm{h} \times \mathrm{v}]$ of $12 \times 16.5 \mu \mathrm{m}^{2}$ FWHM for the Estherville sample was achieved. The energy distribution of the source follows a Lorentzian squared distribution with a linewidth of $0.27 \mathrm{~mm} \mathrm{~s}^{-1}$. The Doppler shift was provided by oscillating the ${ }^{57} \mathrm{FeBO}_{3}$ nuclear monochromator with a sinusoidal drive and maximum velocity of $11.25 \mathrm{~mm} \mathrm{~s}^{-1}$ distributed over 1048 velocity channels (unfolded) in the multi-channel analyzer. All spectra were acquired at room temperature $\left(\sim 22{ }^{\circ} \mathrm{C}\right)$. The storage ring was operated in $7 / 8+1$ filling mode. Samples were mounted over holes in an $\mathrm{Al}$ holder. The holder was bolted to a large moving stage with $\pm 1 \mu \mathrm{m}$ accuracy. Sample navigation was achieved by first mapping changes in X-ray absorption as the sample was rastered across the X-ray beam, enabling the XY stage positions corresponding to prominent features around the sample edge to be determined. The locations of individual spectral measurements were then determined by triangulation of the stage position relative to scanning electron microscope (SEM) images of the samples on the holder. Using this method we estimate the uncertainty in the absolute position of individual spectral measurements to be $\sim 10-20 \mu \mathrm{m}$. The uncertainty in the relative position of spectral measurements within a single profile is equal to the accuracy of the stage movement. Due to the high intensity of synchrotron radiation, average spectrum acquisition time was $\sim 2800$ seconds.

Phase fractions of kamacite, tetrataenite and antitaenite were calculated by comparing the relative proportions of each phase from the Mössbauer spectra and normalising them based on Fe content. Fe contents of kamacite, tetrataenite and antitaenite are assumed to be 95 at.\%, 50 at.\% and 85 at.\%, respectively (Blukis et al., 2017).

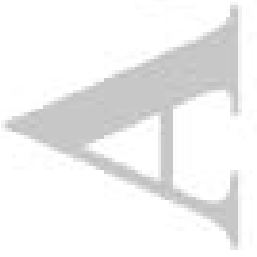




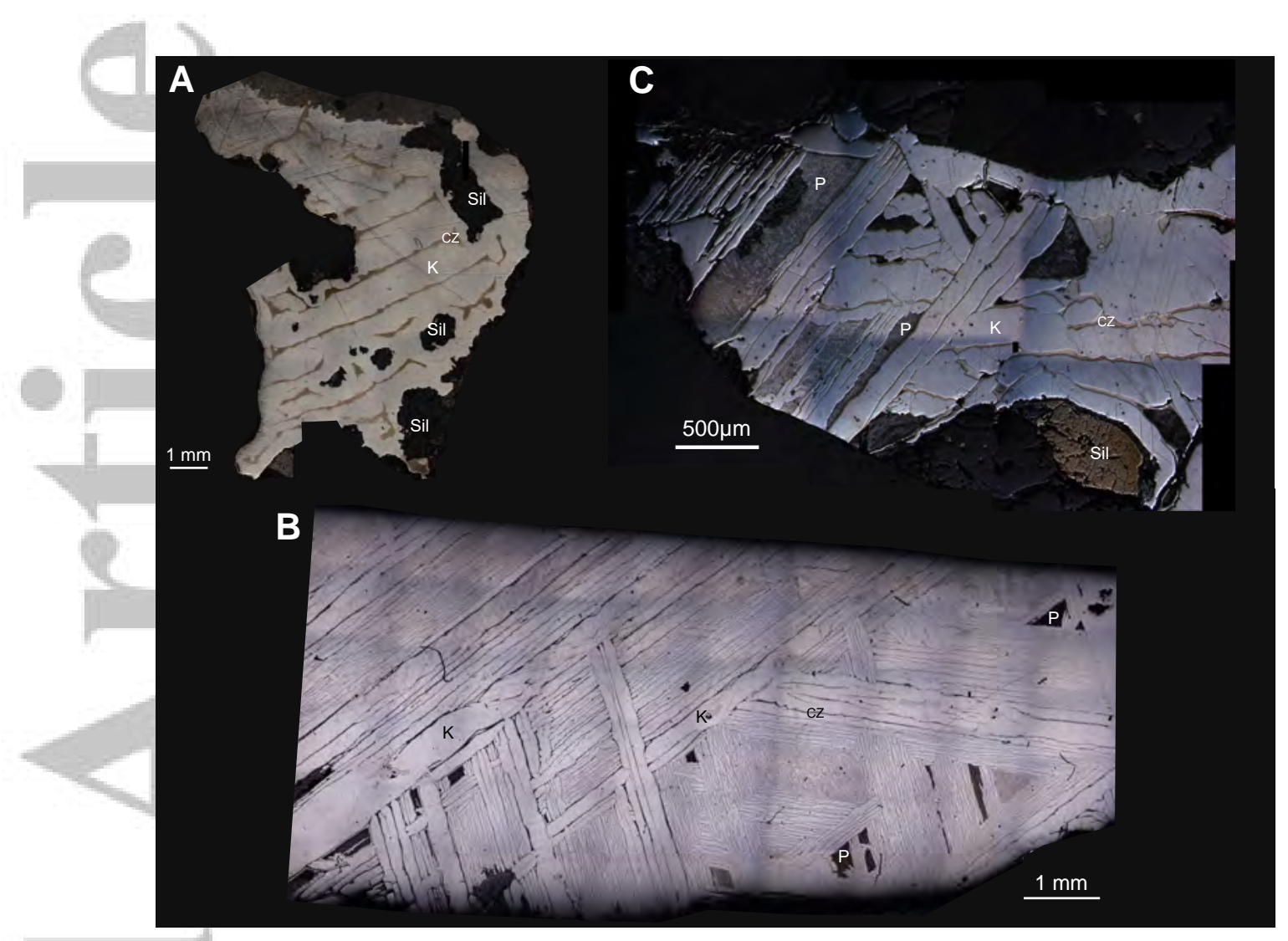

Figure 1: Polished and etched sections of (a) the Estherville mesosiderite $(9.2 \mathrm{wt} . \% \mathrm{Ni})$ (b) the Brenham pallasite (10.6 wt.\% Ni) and (c) the Steinbach IVA iron (9.4 wt.\% Ni). Compositions are from Goldstein et al. (2014). The images highlight the clear changes in lengthscale of the Widmanstätten pattern with cooling rate; Estherville exhibits the slowest cooling rate and the coarsest features, and vice versa for Steinbach. Each image is annotated to highlight kamacite lamellae $(\mathrm{K})$, the cloudy zone $(\mathrm{CZ})$, plessite $(\mathrm{P})$ and silicates (Sil).

\section{Results}

\subsection{Cloudy zone behaviour as a function of cooling rate and proximity to the tetrataenite rim}

The lengthscale on which Fe-Ni microstructures form is dependent on cooling rate and $\mathrm{Ni}$ content. The effect of cooling rate can be observed by examining polished and etched surfaces of iron meteorites with similar Ni contents (Figure 1). In fast-cooled samples the Widmanstätten pattern forms on a much finer scale; the kamacite lamellae are narrow (200 - $300 \mu \mathrm{m}$ in width), as are the Ni-rich regions between them. In the slowest cooled samples the Widmanstätten pattern is much broader (kamacite lamellae have a width of $1-2 \mathrm{~mm}$ ). These variations are 
also observed within the individual FeNi microstructures. In this section we will review how the cloudy zone microstructure varies with cooling rate, focusing on the variations in the resultant magnetic behaviour.

The fastest cooled sample reviewed here is Bishop Canyon, a IVA iron meteorite with a cooling rate of $2500{ }^{\circ} \mathrm{C} \mathrm{Myr}^{-1}$ (Yang et al., 2008). It has a tetrataenite rim with a width of $80 \pm 4 \mathrm{~nm}$ and an average cloudy zone island diameter of $12 \pm 3 \mathrm{~nm}$ (Goldstein et al., 2009b). A map of the magnetization direction within the kamacite, the tetrataenite rim, the cloudy zone and plessite in Bishop Canyon is shown in Figure 2. All four microstructures show multidomain behaviour, however domain size varies between microstructures. The largest domains $(\sim 1 \mu \mathrm{m})$ are observed in the kamacite. The tetrataenite rim is very narrow and magnetic domains alternate along its length approximately once every $1-5 \mu \mathrm{m}$. In the cloudy zone, narrow regions of uniform magnetization (width of $100-200 \mathrm{~nm}$ ) run parallel to the tetrataenite rim. There is no observable distinction between the magnetization of the islands compared to the matrix in the cloudy zone; the orientation and shape of the regions of uniform magnetization appear to only be controlled by the parallel boundaries between the microstructures.

IVA iron meteorites have a large range of cooling rates; Steinbach and Chinautla have much slower cooling histories than Bishop Canyon (150 and $110{ }^{\circ} \mathrm{C} \mathrm{Myr}^{-1}$, respectively). Their microstructures are therefore significantly larger; Steinbach and Chinautla have tetrataenite rim widths of $195 \pm 5 \mathrm{~nm}$ and $215 \pm 25 \mathrm{~nm}$, and cloudy zone island diameters of $29 \pm 3 \mathrm{~nm}$ and $32 \pm 4.5 \mathrm{~nm}$, respectively (Goldstein et al., 2009b). Compositional and magnetization maps of kamacite, the tetrataenite rim, the cloudy zone and plessite are shown for Steinbach in Figure 3. The kamacite shows large magnetic domains $(>1 \mu \mathrm{m})$, consistent with multidomain behaviour. The tetrataenite rim also shows multidomain behaviour, but here the domains are much smaller $(200-300 \mathrm{~nm})$. In the cloudy zone immediately adjacent to the tetrataenite rim, the magnetization pattern appears to cluster around the islands, forming small regions of uniform magnetization $(<200 \mathrm{~nm})$. Further from the tetrataenite rim in the fine cloudy zone, the islands and matrix are uniformly magnetized.

The pallasites and IAB iron meteorites reviewed here have intermediate cooling rates $(7.6-$ 


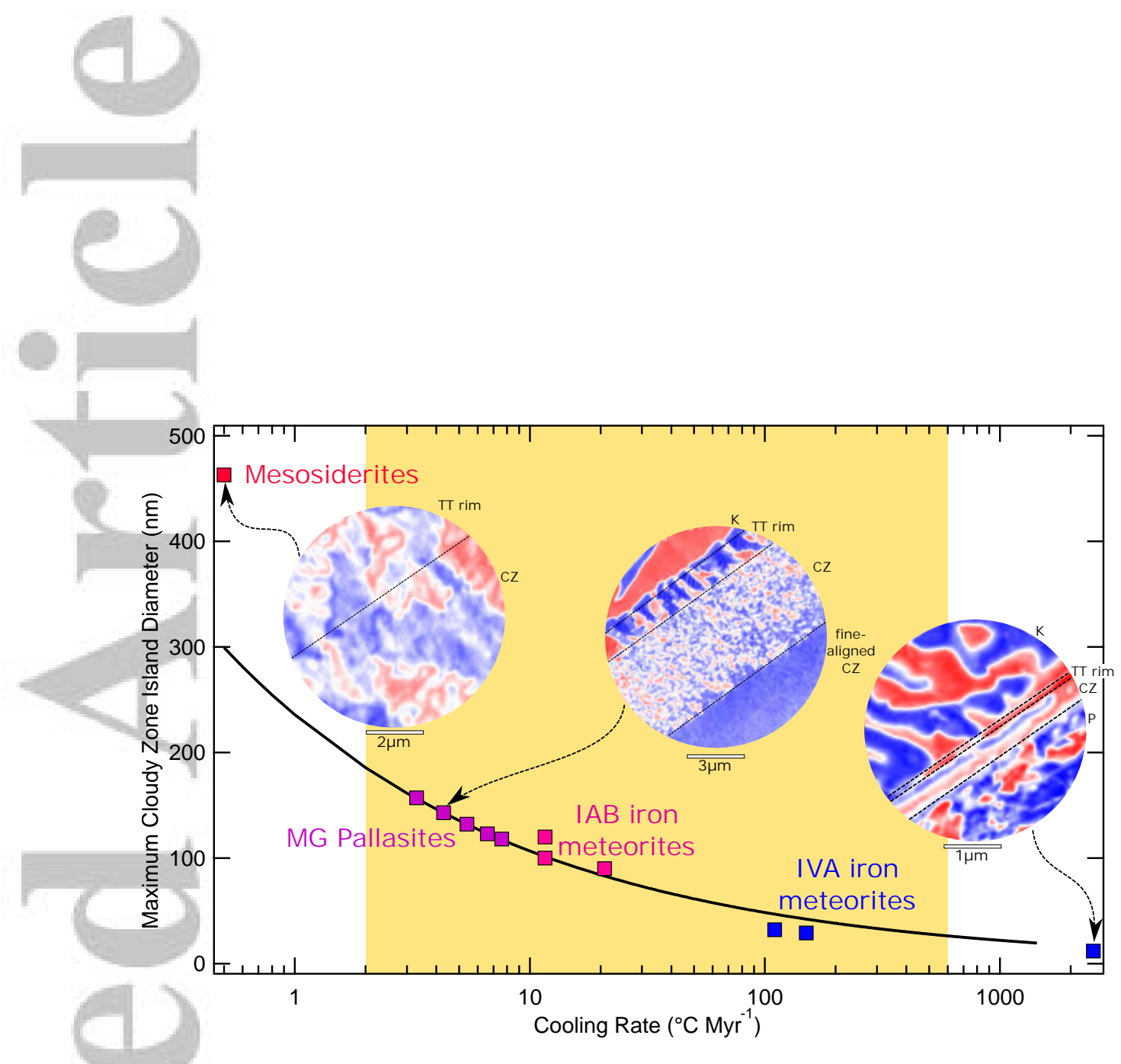

Figure 2: The graph shows the meteorites whose cloudy zone have been studied using XPEEM. The black curve represents the empirically-derived relationship between cloudy zone island diameter and cooling rate defined by Yang et al. (2010). X-PEEM images show the magnetization in the kamacite $(\mathrm{K})$, tetrataenite rim (TT rim), cloudy zone (CZ) and plessite $(\mathrm{P})$ and the clear differences in the magnetic behaviour of these microstructures with cooling rate. The X-PEEM images shown are from the Estherville mesosiderite, the Marjalahti pallasite (Nichols et al., 2016) and the Bishop Canyon IVA iron meteorite (Bryson et al., 2017). The yellow region represents ideal cloudy zone for paleomagnetic study, which and

depends upon both cooling rate and spatial proximity to the tetrataenite rim.

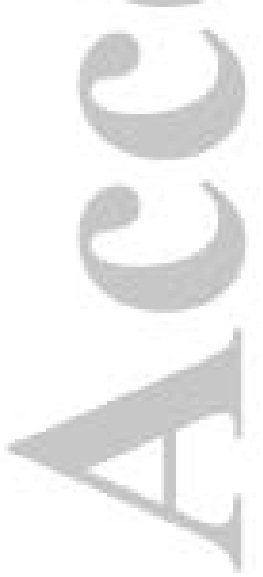




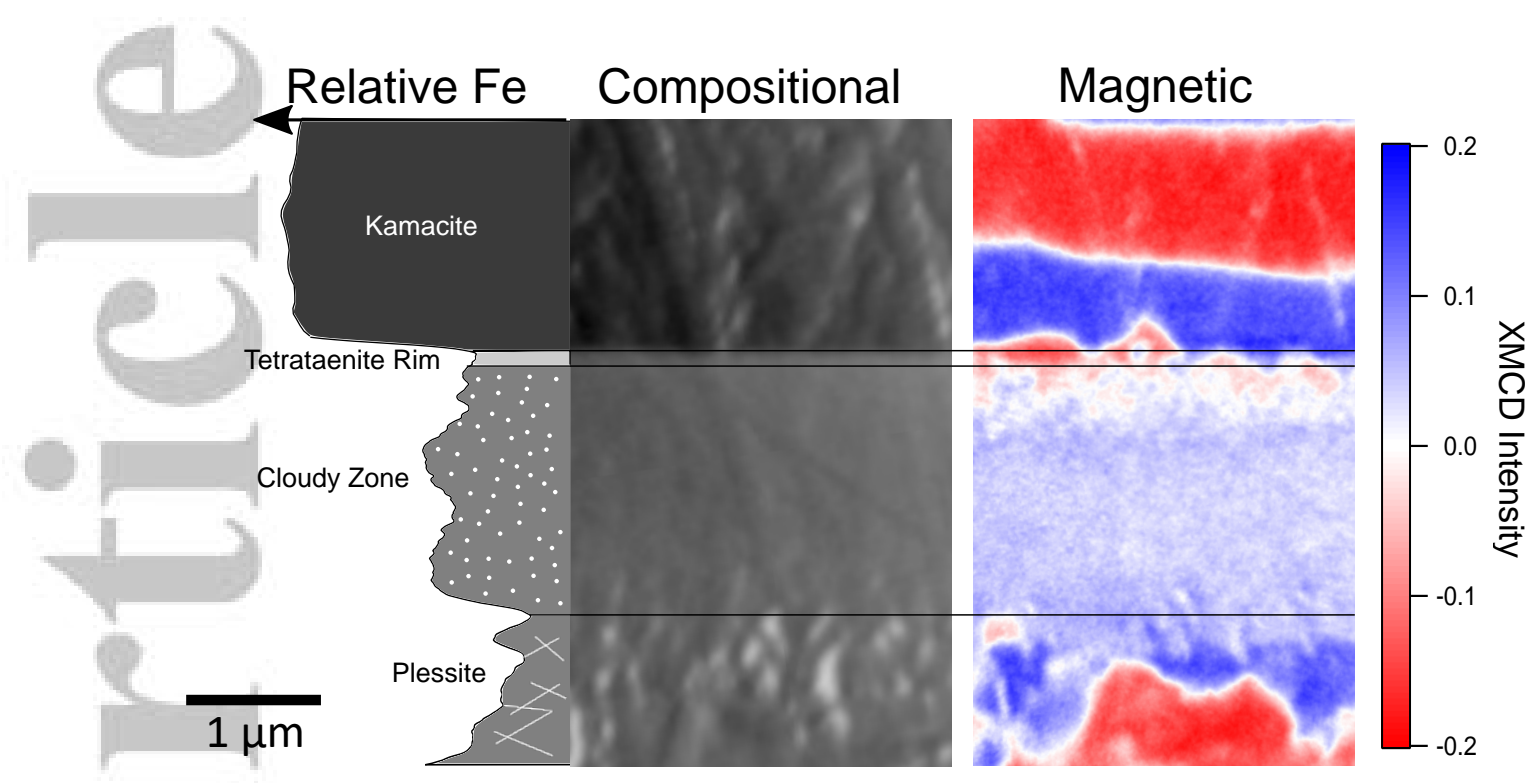

Figure 3: An example of a magnetic and compositional profile across the kamacite, tetrataenite rim, cloudy zone and plessite in the Steinbach IVA iron meteorite (Bryson et al., 2017). Note that the magnetic and compositional images do not correspond to the same region. All of the relevant microstructures were imaged in a single field of view.

$20.8^{\circ} \mathrm{C} \mathrm{Myr}^{-1}$ ) (Yang et al., 2010; Winfield et al., 2012; Goldstein et al., 2014). magnetization maps of the kamacite, the tetrataenite rim and the cloudy zone are shown for Imilac and Brenham in Figures 2 and 4a, respectively. The kamacite and tetrataenite rim both exhibit multidomain magnetic behaviour. Magnetic domains in the kamacite are significantly larger than those in the tetrataenite rim. The cloudy zone within $\sim 4$ widths of the tetrataenite rim is clustered into uniformly magnetized regions on a similar lengthscale to the size of the cloudy zone islands (90 - $160 \mathrm{~nm}$ ) (Yang et al., 2010; Goldstein et al., 2014). In the fine cloudy zone, at distances exceeding $\sim 4$ tetrataenite rim widths the clustering is replaced by a region of uniform magnetization. In some cases, this transition is very sharply defined, such as in the magnetization map of Imilac (Figure 2) whereas the transition is more gradual and happens further from the tetrataenite rim in Brenham (Figure 4a). The sharpness of the boundary between the coarse and fine cloudy zone does not appear to correlate systematically with cooling rate and is also observed to vary within a single sample.

The mesosiderites have the slowest known cooling rates of any meteorite group. Here we present the first characterisation of the magnetic properties of the Estherville mesosiderite 

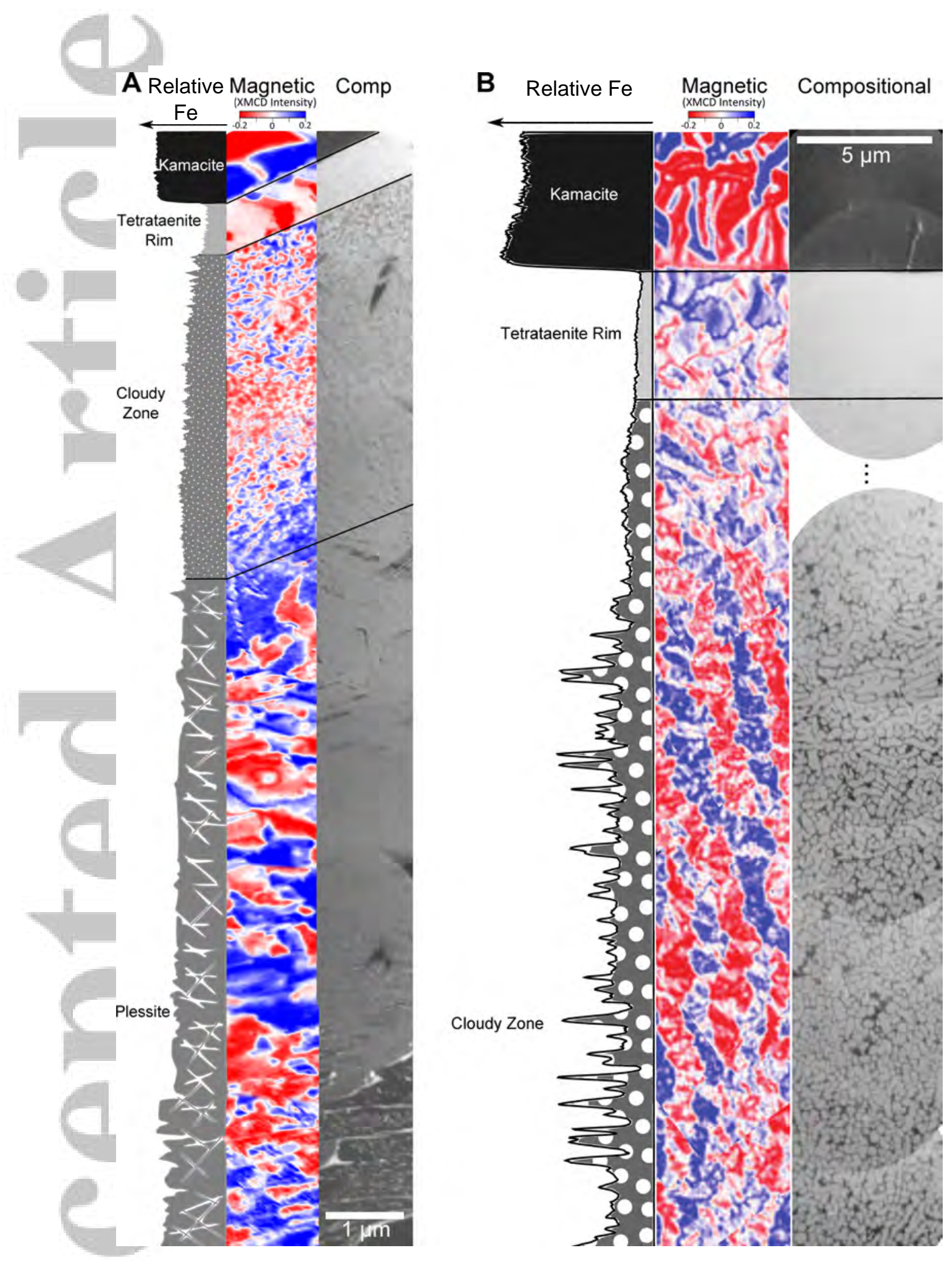

Figure 4: (a) X-PEEM images of the composition and magnetization of microstructures in the Brenham pallasite, with associated compositional profile. Brenham cooled at $\sim 6{ }^{\circ} \mathrm{C} \mathrm{Myr}^{-1}$ below $\sim 500{ }^{\circ} \mathrm{C}$ (Yang et al., 2010). (b) X-PEEM images of the composition and magnetization of microstructures in the Estherville mesosiderite, with associated compositional profile. Estherville cooled at $\sim 0.5{ }^{\circ} \mathrm{C} \mathrm{Myr}^{-1}$ below $\sim 500{ }^{\circ} \mathrm{C}$ (Yang et al., 1997). 
(cooling rate $<0.5{ }^{\circ} \mathrm{C} \mathrm{Myr}^{-1}$ ). magnetization maps of Estherville are shown in Figures 2 and 4b. The microstructures here form on a much coarser scale; the tetrataenite rim width is $\sim 5 \mu \mathrm{m}$ and the average cloudy zone island diameter is $463 \pm 32 \mathrm{~nm}$ (Goldstein et al., 2014). The kamacite has large $(1-2 \mu \mathrm{m}$ width) magnetic domains with a higher XMCD intensity than the magnetization of the tetrataenite rim and the cloudy zone. The tetrataenite rim and the cloudy zone both exhibit multidomain behaviour and there is no clear transition between the two. Large $(\sim 2 \mu \mathrm{m}$ width $)$ regions of uniform magnetization are observed as parallel stripes at a high angle to the kamacite-tetrataenite-rim-interface and run directly across the tetrataenite rim and cloudy zone (Figure 3).

\subsection{Characterising the magnetic properties of the cloudy zone matrix}

The magnetic behaviour of the cloudy zone matrix is not easily examined using X-PEEM; the matrix is typically only a few nm across, and therefore cannot be spatially resolved. Matrix properties are therefore evaluated using Mössbauer spectroscopy to acquire spatially resolved spectral information for each magnetic phase within the cloudy zone. A previous study investigated the behaviour of the cloudy zone in two intermediate cooled samples, Esquel and Tazewell, and showed that their cloudy zones are comprised of ferromagnetic tetrataenite and paramagnetic antitaenite (Blukis et al., 2017). Antitaenite has the same crystallographic structure as taenite, but a different electronic structure resulting in its contrasting magnetic properties (Danon et al., 1979; Rancourt, 1995; Rancourt et al., 1999; Lagarec et al., 2001). Here we compare these results to new data for the slow cooled Estherville mesosiderite.

In all three meteorites, the percentage of the kamacite phase decreases in the cloudy zone. There appears to be a systematic decrease in kamacite content with slower cooling rate, however this is an artifact caused by the larger size of the focussed beam used for Mössbauer measurements compared to the width of the cloudy zone (Blukis et al., 2017). The degree of overlap in the signal between the kamacite and the cloudy zone decreases as the microstructures become larger at lower cooling rates. In Esquel and Tazewell the entire kamacite signal in the cloudy zone is considered to be an artifact; it is solely comprised of antitaenite and tetrataenite. This 
is supported by a scanning precession electron diffraction (SPED) study of Tazewell in which no kamacite was observed in the cloudy zone (Einsle et al., 2018). In Estherville on the other hand, the width of the cloudy zone is significantly larger than the beam size. This suggests there is a genuine kamacite phase component in the Estherville cloudy zone.

It appears that the proportions of antitaenite and tetrataenite in the cloudy zone may vary with cooling rate. In Tazewell and Esquel there are approximately equal proportions of each phase. In Estherville there is significantly less antitaenite in the cloudy zone and the dominant phase is tetrataenite, however the ratio of tetrataenite to kamacite and antitaenite is also approximately equal.

\subsection{Interpreting paleomagnetic signatures in the cloudy zone}

Paleomagnetic information is extracted from the cloudy zone by assessing the degree of bias in magnetization direction in the cloudy zone (Bryson et al., 2014b; Maurel et al., 2019). The cloudy zone adjacent to the tetrataenite rim in two intermediate-cooled samples, Imilac and Toluca, are compared (Figure 6). Given their similarity in island size, $143 \mathrm{~nm}$ and $120 \mathrm{~nm}$ respectively, Imilac and Toluca should acquire a remanence via the same mechanism in this region. The cloudy zone in Imilac shows a strong bias in magnetization direction, whereas Toluca shows no such bias. The bias in magnetization direction in Imilac is attributed to cloudy zone formation in the presence of a strong external field (Bryson et al., 2015). The random distribution of magnetization directions in the cloudy zone in Toluca is attributed to formation in a null or very weak external magnetic field (Nichols et al., 2018). The behaviour of the cloudy zone in this region contrasts starkly with that observed further from the tetrataenite rim (e.g. Brenham pallasite in Figure 4, Steinbach IVA in Figure 3), where relatively uniform magnetization is observed, irrespective of the presence or absence of a bias in cloudy zone closer to the rim. 


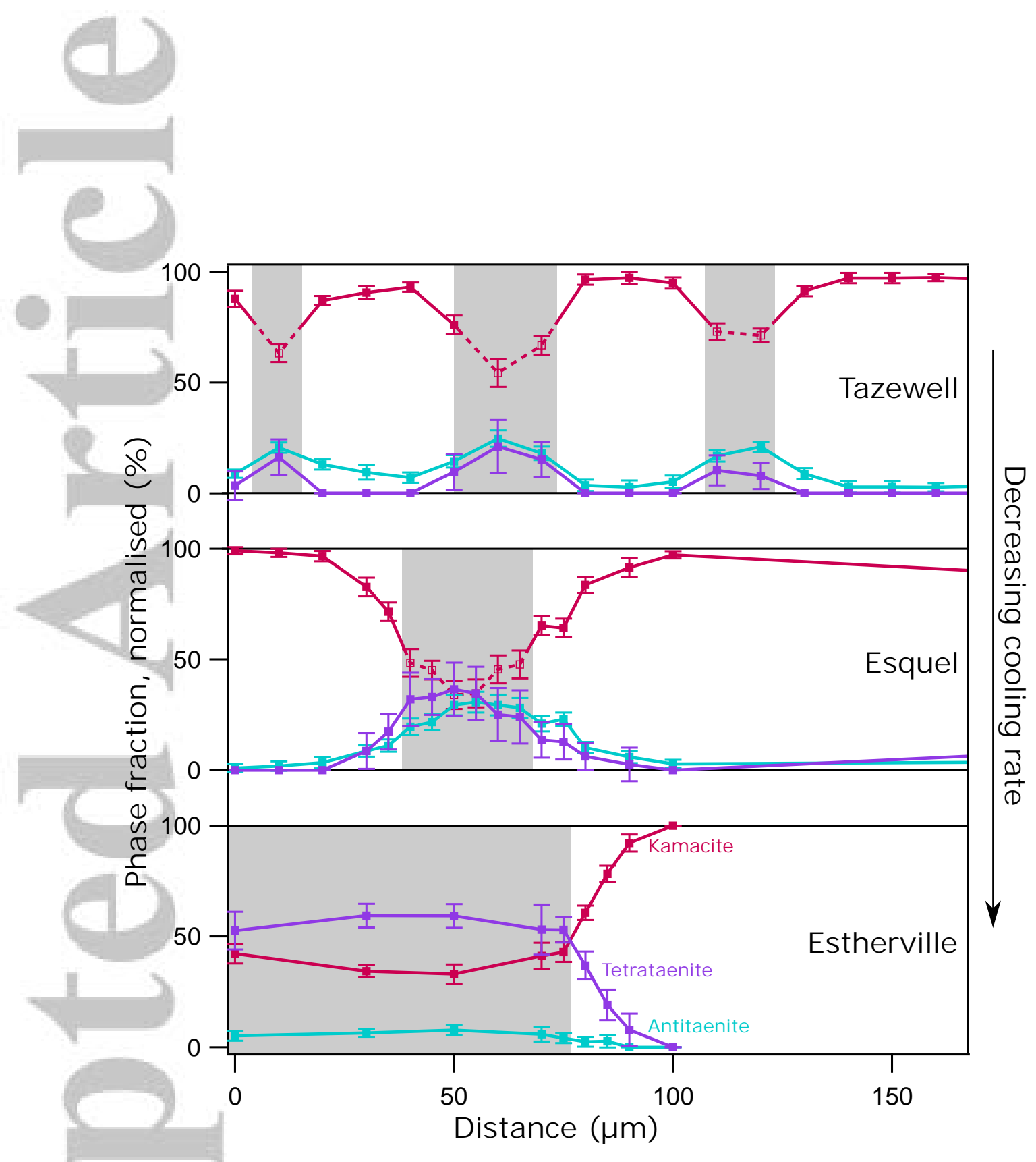

Figure 5: Normalised phase fractions of kamacite, tetrataenite and antitaenite determined from Mössbauer spectra for Tazewell, Esquel and Estherville. Profiles for Tazewell and Esquel were previously published in Blukis et al. (2017). Grey boxes highlight regions of cloudy zone. Dashed lines reflect measurement artifacts; kamacite appears to be present in the cloudy zone of Tazewell and Esquel, however this is only because the beam size is larger than the measured microstructures.

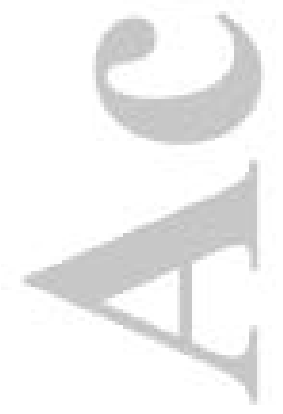




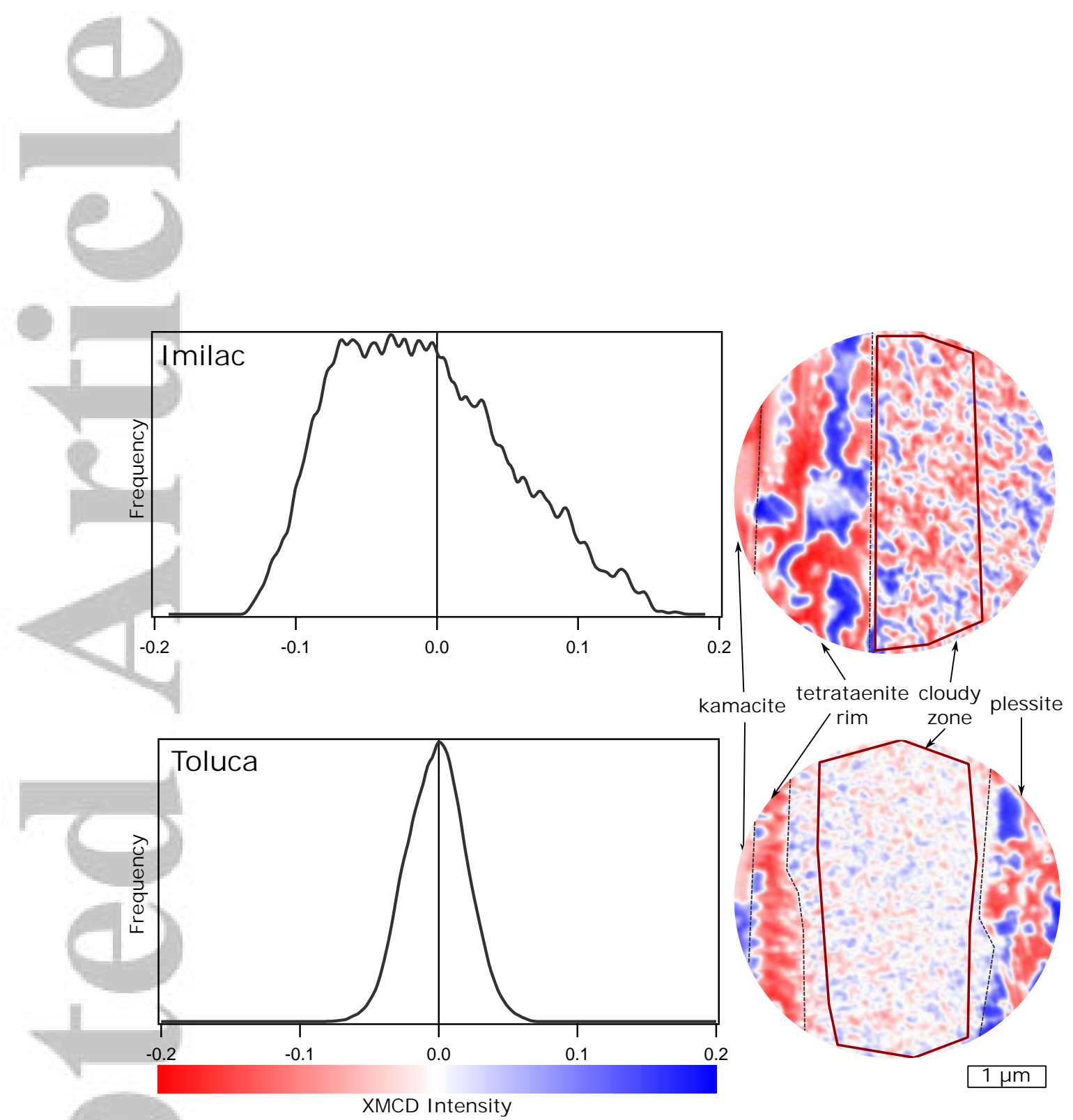

Figure 6: Histograms of the distribution of XMCD pixel intensity in the cloudy zone of Imilac and Toluca. Despite having similar cooling rates and island sizes, these cloudy zones formed under highly contrasting external magnetic fields. Imilac experienced a strong magnetic field shown by the pronounced asymmetric histogram, whereas Toluca experienced a weak or null magnetic field. The regions outlined in dark red were sampled to generate the histograms of pixel intensity. 


\section{Discussion}

\subsection{Variations in cloudy zone behaviour with cooling rate}

The cloudy zone forms via spinodal decomposition in even the fastest cooled IVA iron meteorites, such as the La Grange IVA iron meteorite which cooled at 6,600 ${ }^{\circ} \mathrm{C} \mathrm{Myr}^{-1}$ (Yang et al., 2007, 2008). This suggests that, on meteoritic cooling timescales, spinodal decomposition is always expected to take place to some degree, although the resulting cloudy zone becomes spatially restricted at the fastest cooling rates (Figure 3). Island size decreases with increasing cooling rate (Goldstein et al., 2009a; Yang et al., 2008). We would expect, therefore, to see an increase in the coercivity of the cloudy zone with increasing cooling rate, since the coercivity of tetrataenite has been shown to increase with decreasing lengthscale (Uehara et al., 2011; Bryson et al., 2014a). It is therefore somewhat surprising that Bishop Canyon, the fastest cooled meteorite considered in this study, has a cloudy zone consisting of large regions of uniform magnetization. A study of the hysteresis properties of bulk mental from Bishop Canyon revealed low coercive force, $\mathrm{H}_{c}$, and remanent coercive force, $\mathrm{H}_{c r}$ (Bryson et al., 2017). The measured hysteresis loop was closed at all applied field values, suggesting the bulk metal contains no magnetically hard material. Similar hysteresis properties have been observed in heated samples of Santa Catharina, an ungrouped IAB iron meteorite, and of ordinary chondrites (Dos Santos et al., 2015; Gattacceca et al., 2014). In both these studies, samples were heated in the laboratory to transform tetrataenite islands into taenite via $\mathrm{Fe}-\mathrm{Ni}$ disordering. This $\mathrm{Fe}-\mathrm{Ni}$ disordering causes a drastic change in magnetic properties, including a significant decrease in saturation remanence, $\mathrm{M}_{r s}, \mathrm{H}_{c}$ and $\mathrm{H}_{c r}$ (Gattacceca et al., 2014). Disordering could be caused by shock heating during an impact event since the process only requires short-range (latticescale) diffusion and therefore only heating for short periods of time is required, for example complete disordering take $<100$ seconds at $550{ }^{\circ} \mathrm{C}$ (Goldstein et al., 2009b; Dos Santos et al., 2015). However, the Bristol IVA iron meteorite, which has not experienced significant shock (Yang et al., 2007), has been shown to contain a mixture of ordered and disordered islands using atom probe tomography (Rout et al., 2017). Since Bishop Canyon also only experienced 
low levels of shock ( $<13 \mathrm{GPa}$ ) (Yang et al., 2007) we argue that the cloudy zone is comprised of taenite islands because cooling was too rapid for ordering to tetrataenite to occur in the first place. Because of the low temperature of tetrataenite ordering $\left(<320{ }^{\circ} \mathrm{C}\right)$ kinetics are significantly inhibited and ordering takes at least $10^{4}$ years per atomic jump (Scorzelli, 1997). Given the rapid cooling of Bishop Canyon $\left(2500 \pm 1.3{ }^{\circ} \mathrm{C} \mathrm{Myr}^{-1}\right)$ cooling from $320^{\circ} \mathrm{C}$ to room temperature would take $\sim 120,000$ years allowing insufficient time for complete ordering.

The large uniform regions of magnetization observed in the cloudy zone of Bishop Canyon are not observed in the slower cooled IVA iron meteorites, Steinbach and Chinautla. In these samples, small regions of uniform magnetization are observed to cluster around islands in the cloudy zone near the tetrataenite rim. Similarly their cloudy zones also have different hysteresis properties suggesting the presence of tetrataenite (Bryson et al., 2017; Gattacceca et al., 2014). The different magnetic properties observed in the cloudy zone of Bishop Canyon could be caused by the smaller size of the islands, approaching the superparamagnetic threshold of $\sim 5 \mathrm{~nm}$ (Neel et al., 1964), or insufficient time for tetrataenite ordering. The cloudy zone in Bishop Canyon shows visibly different magnetic behaviour to the fine-aligned cloudy zone in meteorites with intermediate cooling rates, although the islands are comparable in size. We therefore suggest that for cooling rates $<150{ }^{\circ} \mathrm{C} \mathrm{Myr}^{-1}$, there is sufficient time for ordering and cloudy zone islands are formed of tetrataenite, while at cooling rates exceeding $2500{ }^{\circ} \mathrm{C} \mathrm{Myr}^{-1}$ the islands do not order and remain as taenite. We do not observe superparamagnetic behaviour (in which case the cloudy zone would appear unmnagetized on our observation timescales) because this behaviour is likely to be suppressed by interactions with neighbouring particles (Varón et al., 2013) and surface effects (Blukis et al., 2017). The drastic change in magnetic properties associated with this ordering suggests that the fastest cooled meteorites are not able to record paleomagnetic information; this only occurs if tetrataenite forms (Gattacceca et al., 2014; Einsle et al., 2018). Clustering of magnetization around islands in the cloudy zone is observed in all the samples considered here with cooling rates between $7.6-150{ }^{\circ} \mathrm{C} \mathrm{Myr}^{-1}$. We therefore assume that the magnetic properties of the cloudy zone in this range of cooling rates is similar. In the slowest cooled sample considered here, the Estherville mesosiderite, the magneti- 
zation of the cloudy zone is significantly different. This is most likely due to the large size of the tetrataenite islands $(463 \mathrm{~nm})$ that readily permit the formation of magnetic domain walls, resulting in lower coercivities (Uehara et al., 2011; Bryson et al., 2014b). The magnetic behaviour is also likely to be influenced by the fundamentally different chemical composition of the cloudy zone in Estherville (Figure 5). Cooling rates in the mesosiderites are so slow that the cloudy zone undergoes further breakdown; the islands develop Fe-rich interiors and kamacite is observed in the matrix (Yang et al., 1996). We expect the magnetic properties of the cloudy zone to inherently change if the matrix becomes ferromagnetic kamacite, rather than paramagnetic antitaenite which is observed in faster cooled samples (Blukis et al., 2017; Einsle et al., 2018).

\subsection{Ideal cloudy zone for paleomagnetic study}

In this section we will define the region of cloudy zone that reliably records a paleomagnetic remanence. This is controlled by both the thermal history and proximity to other microstructures. Based on the meteorites reviewed in this study, cloudy zone with ideal paleomagnetic properties forms at cooling rates between $7.6-150{ }^{\circ} \mathrm{C} \mathrm{Myr}^{-1}$, bounded at the upper end by the rate needed to form tetrataenite and at the lower end by the need to avoid multi-domain islands. A recent study of the Tazewell IAB iron meteorite (cooling rate $20.8^{\circ} \mathrm{C} \mathrm{Myr}^{-1}$ ) combined high-resolution microscopy and micromagnetic simulations to demonstrate how paleomagnetic signals are acquired by the cloudy zone (Einsle et al., 2018). Islands in the coarse and mid cloudy zone form as single-vortex taenite. Upon ordering to tetrataenite at temperatures below $320{ }^{\circ} \mathrm{C}$ the vortex transforms to a transient two-domain state, with a discrete $180^{\circ}$ domain wall separating two oppositely magnetized domains. This transformation is attributed to the increase in uniaxial anisotropy induced by Fe-Ni ordering. Strong magnetostatic interaction between neighbouring islands causes the domain wall to displace until it reaches the edge of the island, where it denucleates permanently. The interaction-driven domain-state transition from a two-domain to single-domain leaves each island uniformly magnetized either parallel or antiparallel to its crystallographically defined easy axis (dictated by the crystallographic 
orientation of $\mathrm{Fe}-\mathrm{Ni}$ ordering). In the absence of an applied magnetic field, the distribution of interaction fields amongst the closely packed islands is random and centred around zero. Random magnetostatic interactions will lead to equal probability of domain walls displacing one way or the other, resulting in a cloudy zone of single-domain islands magnetized randomly along each of the six possible easy axis directions (e.g., Figure 6b). An applied field, however, biases the interaction field distribution, which is no longer centered around zero, leading to a cloudy zone of single-domain islands that are preferentially magnetized along whichever of the six possible easy axes is closest to the applied field (e.g., Figure 6a). In this model, the presence of the vortex state is the defining characteristic of cloudy zone suitable for paleomagnetic study; it provides a nucleation point for the two-domain state, which in turn provides the mechanism by which the magnetization state of an island can be influenced by an applied field.

For spherical taenite particles at $320^{\circ} \mathrm{C}$ the single-domain to vortex transition size is $20-$ $25 \mathrm{~nm}$ (Einsle et al., 2018). Therefore, any taenite islands that are smaller than this threshold will adopt single-domain states rather than vortex states, with consequences for both the mechanism of remanence acquisition and the strength of magnetostatic interactions between the islands. A critical observation here is that typical 'coarse cloudy zone' behaviour is restricted to a very narrow region immediately next to the tetrataenite rim in both the Steinbach and Chinautla IVA meteorites (Figure 3 and Bryson et al. (2017)). These meteorites have island diameters adjacent to the tetrataenite rim of $29 \pm 3 \mathrm{~nm}$ and $32 \pm 4.5 \mathrm{~nm}$, respectively (Goldstein et al., 2009b), which are both just above the threshold size for vortex behaviour. This observation supports the hypothesis that the adoption of vortex states is a prerequisite for generating 'coarse cloudy zone' behaviour of the type that is able to respond to the presence or absence of a paleofield in the manner displayed in Figure 6. Islands just a short distance away from the tetrataenite rim in the Steinbach and Chinautla meteorites fall below the $20-25 \mathrm{~nm}$ threshold and would have adopted single-domain states.

We observe such variations in the magnetization state of the cloudy zone with distance from the tetrataenite rim in all meteorites with cooling rates between $7.6-150{ }^{\circ} \mathrm{C} \mathrm{Myr}^{-1}$. The coarse cloudy zone, immediately adjacent to the tetrataenite rim, exhibits clustering of 
uniform magnetization around the tetrataenite islands. The fine cloudy zone, furthest from the tetrataenite rim exhibits strikingly different behaviour; large patches are uniformly magnetized and it is referred to as the 'fine-aligned' cloudy zone (Harrison et al., 2017). We propose that the absence of precursor vortex states in the fine cloudy zone leads to both the increased magnetostatic interactions and the lack of a mechanism to enable the magnetization state to be influence by the applied field. The result is a high degree of spontaneous magnetization that no longer carries useful information about the paleofield.

As Ni content decreases away from the tetrataenite rim, the spinodal initiation temperature also decreases (Maurel et al., 2019). An important transition may occur when the spinodal initiation temperature drops below $320^{\circ} \mathrm{C}$, at which point islands will form as tetrataenite rather than taenite. According to current knowledge of the Fe-Ni phase diagram, this point is reached when the $\mathrm{Ni}$ composition is bewteen $\sim 34-41$ wt\% Ni (Maurel et al., 2019; Yang et al., 1997). The uniaxial anisotropy of tetrataenite means that two-domain states or single-domain states, rather than vortex states, are formed when islands grow, with the single-domain to twodomain threshold size being $50-55 \mathrm{~nm}$, roughly twice that the taenite single-domain to vortex threshold (Einsle et al., 2018). Therefore, islands that grow as tetrataenite are more likely to be single domain than equivalently sized taenite particles. Therefore, the limit of the reliable paleomagnetic remanence acquisition is defined either by the condition $\mathrm{Ni}>34-40 \mathrm{wt} \%$ and island sizes $<20-25 \mathrm{~nm}$, or $\mathrm{Ni}<34-40 \mathrm{wt} \%$ and island sizes $<50-55 \mathrm{~nm}$, whichever is reached sooner.

Magnetostatic interactions do not have a significant influence on cloudy zone suitable for paleomagnetic study. This is supported by the fact that cloudy zone which forms in the absence of an external magnetic field shows a random distribution of magnetization directions (Figure $6)$. 


\section{Conclusions}

The formation of Fe-Ni microstructures and their corresponding magnetic properties have been reviewed for four groups of meteorites spanning a large range of cooling rates; the mesosiderites, the IAB iron meteorites, the pallasites and the IVA iron meteorites. We identify the optimal conditions that lead to the generation of cloudy zone with the best potential to acquire nanopaleomagnetic remanence:

- Islands ideally form within the taenite stability field at $\mathrm{T}>320{ }^{\circ} \mathrm{C}$ and have sizes $>20$ $25 \mathrm{~nm}$, such that they are able to adopt a precursor single-vortex micromagnetic state.

- Island sizes decrease with increasing distance from the tetrataenite rim, so that only islands that are sufficiently close to the rim are above the $20-25 \mathrm{~nm}$ threshold.

- Spinodal decomposition occurs at lower temperatures with increasing distance from the tetrataenite rim, so that only islands that are sufficiently close to the rim will have formed above $320{ }^{\circ} \mathrm{C}$.

- Cooling rates must be slow enough $\left(\lesssim 150-2500{ }^{\circ} \mathrm{C} \mathrm{Myr}^{-1}\right)$ to allow islands to transform to tetrataenite on cooling below $320^{\circ} \mathrm{C}$, thereby inducing a domain state transition from vortex to two-domain to single-domain. This mechanism enables the cloudy zone magnetization to respond to the paleofield, creating a bias that can be detected using X-PEEM.

- Cooling rates must be fast enough $\left(\gtrsim 1^{\circ} \mathrm{C} \mathrm{Myr}{ }^{-1}\right)$ to prevent islands growing too large and creating multi-domain behaviour.

- Mesosiderites (cooling rate $\lesssim 0.5-3.3{ }^{\circ} \mathrm{C} \mathrm{Myr}{ }^{-1}$ ): Cloudy zone is multidomain and does not acquire a stable paleomagnetic remanence, possibly due to large island size or the composition and mineralogy of the matrix.

- Pallasites and IAB iron meteorites (cooling rate $\sim 1-100{ }^{\circ} \mathrm{C} \mathrm{Myr}{ }^{-1}$ ): Cloudy zone is a reliable paleomagnetic recorder (Bryson et al., 2015; Nichols et al., 2016, 2018) and 
remanence is acquired during tetrataenite formation. Ordering occurs simultaneously across the cloudy zone, therefore no time-resolved paleomagnetic signals are preserved.

- Intermediate cooled IVA iron meteorites (cooling rate $100-500{ }^{\circ} \mathrm{C} \mathrm{Myr}^{-1}$ ): Only regions of cloudy zone very close to the tetrataenite rim are large enough to form precursor vortex states (Bryson et al., 2017).

- Fastest cooled IVA iron meteorites (cooling rate $>150-2500{ }^{\circ} \mathrm{C} \mathrm{Myr}^{-1}$ ): Cloudy zone forms too quickly for the islands to order to form tetrataenite.

\section{Acknowledgements}

We thank Jérôme Gattacceca and an anonymous reviewer for helpful comments that improved the manuscript. This work was supported by the European Research Council under the European Union's Seventh Framework Programme (FP/2007-2013)/ ERC grant agreement numbers 320750 and 312284. We acknowledge the Helmholtz-Zentrum Berlin for the use of the synchrotron radiation beam time at beamline UE49 of BESSY II. We acknowledge the European Synchrotron Radiation Facility in Grenoble, France for use of synchrotron radiation beam time at the Nuclear Resonance beamline ID18. We thank the Sedgwick Museum, University of Cambridge and the Natural History Museum, London for samples. All data associated with this study are available on zenodo (https://doi.org/10.5281/zenodo.3613625). 


\section{References}

G. K. Benedix, T. J. McCoy, K. Keil, and S. G. Love. A petrologic study of the IAB iron meteorites: Constraints on the formation of the IAB-Winonaite parent body. Meteoritics \& Planetary Science, 35:1127-1141, 2000. ISSN 10869379. doi: 10.1111/j.1945-5100.2000.tb01502.x.

G.K. Benedix, H. Haack, and T.J. McCoy. Iron and Stony-Iron Meteorites. Treatise on Geochemistry, 1:267-285, 2014. doi: 10.1016/B978-0-08-095975-7.00109-1. URL http://linkinghub.elsevier.com/retrieve/pii/B9780080959757001091.

Roberts Blukis, Rudolf Rüffer, Aleksandr I. Chumakov, and Richard J. Harrison. A high spatial resolution synchrotron Mössbauer study of the Tazewell IIICD and Esquel pallasite meteorites. Meteoritics \& Planetary Science, 52(5):925-936, 2017. ISSN 10869379. doi: $10.1111 /$ maps. 12841.

Donald D. Bogard and Daniel H. Garrison. 39Ar - 40Ar ages and thermal history of mesosiderites. Geochimica et Cosmochimica Acta, 62(8):1459-1468, 1998.

James F J Bryson, Claire I O Nichols, Julia Herrero-Albillos, Florian Kronast, Takeshi Kasama, Hossein Alimadadi, Gerrit van der Laan, Francis Nimmo, and Richard J Harrison. Long-lived magnetism from solidification-driven convection on the pallasite parent body. Nature, 517(7535):472-475, 2015. ISSN 0028-0836. doi: 10.1038/nature14114. URL http://dx.doi.org/10.1038/nature14114.

James F.J. Bryson, Nathan S. Church, Takeshi Kasama, and Richard J. Harrison. Nanomagnetic intergrowths in FeNi meteoritic metal. The potential for time resolved records of planetesimal dynamo fields. Earth and Planetary Science Letters, 388: 237-248, feb 2014a. ISSN 0012821X. doi: 10.1016/j.epsl.2013.12.004. URL http://linkinghub.elsevier.com/retrieve/pii/S0012821X13007012.

James F.J. Bryson, Julia Herrero-Albillos, Florian Kronast, Massimo Ghidini, Simon A.T. Redfern, Gerrit van der Laan, and Richard J. Harrison. Nanopaleomagnetism of meteoritic FeNi studied using Xray photoemission electron microscopy. Earth and Planetary Science Letters, 396:125-133, 2014b. ISSN 0012821X. doi: 10.1016/j.epsl.2014.04.016. URL http://www.sciencedirect.com/science/article/pii/S0012821X14002489.

James F.J. Bryson, Benjamin P. Weiss, Richard J. Harrison, Julia Herrero-Albillos, and Florian Kronast. Paleomagnetic evidence for dynamo activity driven by inward crystallisation of a metallic asteroid. Earth and Planetary Science Letters, 472:152-163, 2017. ISSN 0012821X. doi: 10.1016/j.epsl.2017.05.026. URL http://dx.doi.org/10.1016/j .epsl. 2017.05.026.

Vagn F Buchwald. Handbook of Iron Meteorites. University of California Press, 1975.

J Danon, Rosa Bernstein Scorzelli, I Souza Azevedo, W Curvello, JF Albertsen, and JM Knudsen. Iron-nickel 50-50 superstructure in the Santa Catharina meteorite. Nature, 277(January): 10-11, 1979.

Jeremy S. Delaney, C E Nehru, and M Prinz. Olivine clasts from mesosiderites and howardites: Clues to the nature of achondritic parent bodies. Lunar and Planetary Science Conference, 11:1073-1087, 1980. 
E. Dos Santos, J. Gattacceca, P. Rochette, G. Fillion, and R. B. Scorzelli. Kinetics of tetrataenite disordering. Journal of Magnetism and Magnetic Materials, 375:234-241, 2015. ISSN 03048853. doi: 10.1016/j.jmmm.2014.09.051. URL http://dx.doi.org/10.1016/j.jmmm.2014.09.051.

Joshua F. Einsle, Alexander S. Eggeman, Benjamin H. Martineau, Zineb Saghi, Sean M. Collins, Roberts Blukis, Paul A. J. Bagot, Paul A. Midgley, and Richard J. Harrison. Nanopaleomagnetic properties of the meteorite cloudy zone. Proceedings of the National Academy of Sciences, XXX (Xx), 2018. ISSN 0027-8424. doi: 10.1073/pnas.XXXXXXXXXX. URL http://arxiv.org/abs/1803.11502.

Jibamitra Ganguly, H Yang, and S Ghose. Thermal history of mesosiderites: Quantitative constraints from compositional zoning and Fe-Mg ordering in orthopyroxenes. Geochimica et Cosmochimica Acta, 58(12):2711-2723, 1994.

J. Gattacceca, C. Suavet, P. Rochette, B. P. Weiss, M. Winklhofer, M. Uehara, and Jon M. Friedrich. Metal phases in ordinary chondrites: Magnetic hysteresis properties and implications for thermal history. Meteoritics $\&$ Planetary Science, 49(4):652-676, apr 2014. ISSN 10869379. doi: 10.1111/maps.12268. URL http://doi .wiley.com/10.1111/maps.12268.

J I Goldstein and J R Michael. The formation of plessite in meteoritic metal. Meteoritics 86 Planetary Science, 41(4):553-570, 2006.

J. I. Goldstein, J. Yang, P. G. Kotula, J. R. Michael, and E. R. D. Scott. Thermal histories of IVA iron meteorites from transmission electron microscopy of the cloudy zone microstructure. Meteoritics \&f Planetary Science, 44(3):343-358, mar 2009a. ISSN 10869379. doi: 10.1111/j.1945-5100.2009.tb00737.x. URL http://doi.wiley.com/10.1111/j.1945-5100.2009.tb00737.x.

J. I. Goldstein, J. Yang, P. G. Kotula, J. R. Michael, and E. R. D. Scott. Thermal histories of IVA iron meteorites from transmission electron microscopy of the cloudy zone microstructure. Meteoritics \&6 Planetary Science, 44(3):343-358, mar 2009b. ISSN 10869379. doi: 10.1111/j.1945-5100.2009.tb00737.x. URL http://doi.wiley.com/10.1111/j.1945-5100.2009.tb00737.x.

Joseph I. Goldstein, Jijin Yang, and Edward R.D. Scott. Determining cooling rates of iron and stony-iron meteorites from measurements of $\mathrm{Ni}$ and Co at kamacite taenite interfaces. Geochimica et Cosmochimica Acta, 140: 297-320, 2014. ISSN 00167037. doi: 10.1016/j.gca.2014.05.025. URL http://linkinghub.elsevier.com/retrieve/pii/S0016703714003548.

Joseph I. Goldstein, Gary R. Huss, and Edward R.D. Scott. Ion microprobe analyses of carbon in $\mathrm{Fe}-\mathrm{Ni}$ metal in iron meteorites and mesosiderites. Geochimica et Cosmochimica Acta, 200:367-407, 2017. ISSN 00167037. doi: 10.1016/j.gca.2016.12.027. URL http://dx.doi.org/10.1016/j.gca.2016.12.027.

Richard C. Greenwood, Jean Alix Barrat, Edward R.D. Scott, Henning Haack, Paul C. Buchanan, Ian A. Franchi, Akira Yamaguchi, Diane Johnson, Alex W.R. Bevan, and Thomas H. Burbine. Geochemistry and oxygen isotope composition of maingroup pallasites and olivine-rich clasts in mesosiderites: Implications for the "Great 
Dunite Shortage" and HED-mesosiderite connection. Geochimica et Cosmochimica Acta, 169:115-136, 2015. ISSN 00167037. doi: 10.1016/j.gca.2015.07.023. URL http://dx.doi.org/10.1016/j.gca.2015.07.023.

R. J. Harrison, James F J Bryson, C. I. O. Nichols, and B.P. Weiss. Magnetic Mineralogy of Meteoritic Metal : Paleomagnetic Evidence for Dynamo Activity on Differentiated Planetesimals. In Linda T Elkins-Tanton and Benjamin P. Weiss, editors, Planetesimals: Early Differentiation and Consequences for Planets, chapter 10, pages 204-223. Cambridge University Press, 2017. ISBN 9781316339794. doi: 10.1017/9781316339794. URL https://www . cambridge.org/core/books/planetesimals/BAB5DC62F7B5911C96E0DA6C25419CBB.

K. Lagarec, D. G. Rancourt, S. K. Bose, B. Sanyal, and R. A. Dunlap. Observation of a composition-controlled high-moment/low-moment transition in the face centered cubic Fe-Ni system: Invar effect is an expansion, not a contraction. Journal of Magnetism and Magnetic Materials, 236(1-2):107-130, 2001. ISSN 03048853. doi: 10.1016/S0304-8853(01)00449-8.

Clara Maurel, Benjamin P. Weiss, and James F.J. Bryson. Meteorite cloudy zone formation as a quantitative indicator of paleomagnetic field intensities and cooling rates on planetesimals. Earth and Planetary Science Letters, 513:166-175, 2019. ISSN 0012821X. doi: 10.1016/j.epsl.2019.02.027. URL https://doi.org/10.1016/j.epsl.2019.02.027.

David W. Mittlefehldt. The composition of mesosiderite olivine clasts and implications for the origin of pallasites. Earth and Planetary Science Letters, 51:29-40, 1980. ISSN 0012821X. doi: $10.1016 / 0012-821 \mathrm{X}(80) 90254-\mathrm{X}$.

L. Neel, J. Pauleve, R. Pauthenet, J. Laugier, and D. Dautreppe. Magnetic Properties of an Iron-Nickel Single Crystal Ordered by Neutron Bombardment. Journal of Applied Physics, 35(3):873, 1964. ISSN 00218979. doi: 10.1063/1.1713516. URL http://link. aip.org/link/JAPIAU/v35/i3/p873/s1\&Agg=doi.

Jerome A. Neufeld, James F.J. Bryson, and Francis Nimmo. The Top-Down Solidification of Iron Asteroids Driving Dynamo Evolution. Journal of Geophysical Research: Planets, 124 (5):1331-1356, 2019. ISSN 21699100. doi: 10.1029/2018JE005900.

Claire I O Nichols, James F.J. Bryson, Julia Herrero-Albillos, Florian Kronast, Francis Nimmo, and Richard J. Harrison. Pallasite Paleomagnetism: Quiescence of a Core Dynamo. Earth and Planetary Science Letters, 441:103-112, 2016.

Claire I.O. Nichols, BL Getzin, Benjamin Paul Weiss, R. A. Wells, and H. H. Schmitt. The Paleoinclination and Paleointensity of the Early Lunar Dynamo. In AGU Fall Meeting Abstracts, 2018.

H. Ohldag, T. Regan, J. Stöhr, a. Scholl, F. Nolting, J. Lüning, C. Stamm, S. Anders, and R. White. Spectroscopic Identification and Direct Imaging of Interfacial Magnetic Spins. Physical Review Letters, 87(24):247201, nov 2001. ISSN 0031-9007. doi: 10.1103/PhysRevLett.87.247201. URL http://link.aps.org/doi/10.1103/PhysRevLett.87.247201.

Vasily Potapkin, Aleksandr I. Chumakov, Gennadii V. Smirnov, Jean Philippe Celse, Rudolf Rüffer, Catherine McCammon, and Leonid Dubrovinsky. The 57 Fe Synchrotron Mössbauer 
Source at the ESRF. Journal of Synchrotron Radiation, 19(4):559-569, 2012. ISSN 09090495. doi: $10.1107 /$ S0909049512015579.

D G Rancourt. Low-spin Fe-Ni proposed as a new mineral. Journal of Magnetism and Magnetic Materials, 150(95), 1995.

D. G. Rancourt, K. Lagarec, A. Densmore, R. A. Dunlap, J. I. Goldstein, R. J. Reisener, and R. B. Scorzelli. Experimental proof of the distinct electronic structure of a new meteoritic Fe Ni alloy phase. Journal of Magnetism and Magnetic Materials, 191(3):255-260, 1999. ISSN 03048853. doi: 10.1016/S0304-8853(98)00366-7.

Surya S. Rout, Philipp R. Heck, Dieter Isheim, Thomas Stephan, Nestor J. Zaluzec, Dean J. Miller, Andrew M. Davis, and David N. Seidman. Atom-probe tomography and transmission electron microscopy of the kamacite-taenite interface in the fast-cooled Bristol IVA iron meteorite. Meteoritics and Planetary Science, 52(12):2707-2729, 2017. ISSN 10869379. doi: $10.1111 /$ máps. 12988.

Rudolf Rüffer and Aleksandr I. Chumakov. Nuclear Resonance Beamline at ESRF. Hyperfine Interactions, 97-98(1):589-604, 1996. ISSN 0304-3834. doi: 10.1007/BF02150199. URL http://link. springer.com/10.1007/BF02150199.

Alex Ruzicka, William V. Boynton, and Jibamitra Ganguly. Olivine coronas, metamorphism, and the thermal history of the Morristown and Emery mesosiderites. Geochimica et Cosmochimica Acta, 58(12):2725-2741, 1994. ISSN 00167037. doi: 10.1016/0016-7037(94)901406.

T. Schulz, D. Upadhyay, C. Münker, and K. Mezger. Formation and exposure history of nonmagmatic iron meteorites and winonaites: Clues from $\mathrm{Sm}$ and W isotopes. Geochimica et Cosmochimica Acta, 85:200-212, 2012. ISSN 00167037. doi: 10.1016/j.gca.2012.02.012.

RB Scorzelli. A study of phase stability in invar $\mathrm{Fe}-\mathrm{Ni}$ alloys obtained by non-conventional methods. Hyperfine interactions, 110:143-150, 1997. URL http://link.springer.com/article/10.1023/A:1012679517295.

Edward R. D. Scott, Henning Haack, and Stanley G. Love. Formation of Mesosiderites: Fragmentation and Reaccretion of a Large Differentiated Asteroid. Meteoritics and Planetary Science, 36:869-881, 2001. ISSN 10869379. doi: 10.1111/j.1945-5100.2001.tb01927.x.

G.V. Smirnov, U. van Bürck, A.I. Chumakov, A.Q.R. Baron, and R. Rüffer. Synchrotron Mössbauer Source. Physical Review B, 55(9):5811-5815, 1997. ISSN 01631829. doi: 10.1103/PhysRevB.55.5811.

J. Stöhr, H. A. Padmore, S. Anders, T. Stammler, and M. R. Scheinfein. Principles of x-ray magnetic dichroism spectromicroscopy. Surface review and letters, 5(6):1297-1308, 1998.

John a Tarduno, Rory D Cottrell, Francis Nimmo, Julianna Hopkins, Julia Voronov, Austen Erickson, Eric Blackman, Edward R D Scott, and Robert McKinley. Evidence for a dynamo in the main group pallasite parent body. Science, 338(6109):939-942, nov 2012. ISSN 1095-9203. doi: 10.1126/science.1223932. URL http://www.ncbi.nlm.nih.gov/pubmed/23161997. 
Minoru Uehara, Jérôme Gattacceca, Hugues Leroux, Damien Jacob, and Cornelis Jacominus van der Beek. Magnetic microstructures of metal grains in equilibrated ordinary chondrites and implications for paleomagnetism of meteorites. Earth and Planetary Science Letters, 306:241-252, jun 2011. ISSN 0012821X. doi: 10.1016/j.epsl.2011.04.008. URL http://linkinghub.elsevier.com/retrieve/pii/S0012821X11002147.

M. Varón, M. Beleggia, T. Kasama, R. J. Harrison, R. E. Dunin-Borkowski, V. F. Puntes, and C. Frandsen. Dipolar magnetism in ordered and disordered low-dimensional nanoparticle assemblies. Scientific Reports, 3:3-7, 2013. ISSN 20452322. doi: 10.1038/srep01234.

John T. Wasson and Hoppe. Co/Ni double ratios in mesosiderite metal and the unrealistically low cooling rates. 77th Annual Meteoritical Society Meeting, (5405):15-16, 2014. ISSN 10869379. doi: 10.1016/j.molstruc.2014.05.049.

T.B. Winfield, J.I. Goldstein, and E.R.D. Scott. Cooling rate estimates for IAB and IIICD iron meteorites. 43rd Lunar and Planetary Science Conference, (1307):43-44, 2012.

C Yang, D Williams, and J Goldstein. A revision of the Fe-Ni Phase Diagram at Low Temperatures (<400 C). Journal of Phase Equilibria, 17(6):522-531, 1996.

C.W. Yang, D.B. Williams, and J.I. Goldstein. A new empirical cooling rate indicator for meteorites based on the size of the cloudy zone of the metallic phases. Meteoritics \& Planetary Science, 429:423-429, 1997. ISSN 10869379. URL http://onlinelibrary.wiley.com/doi/10.1111/j.1945-5100.1997.tb01285.x/abstract.

Jijin Yang, Joseph I Goldstein, and Edward $\mathrm{R} D$ Scott. Iron meteorite evidence for early formation and catastrophic disruption of protoplanets. Nature, 446 (7138):888-91, apr 2007. ISSN 1476-4687. doi: 10.1038/nature05735. URL http://www.ncbi.nlm.nih.gov/pubmed/17443181.

Jijin Yang, Joseph I. Goldstein, and Edward R.D. Scott. Metallographic cooling and origin of IVA iron meteorites. Geochimica et Cosmochimica Acta, 70 (12):3197-3215, 2008. ISSN 00167037. doi: 10.1016/j.gca.2006.04.007. URL http://www.sciencedirect.com/science/article/pii/S0016703706001888\%5Cnhttp://linkinghu

Jijin Yang, Joseph I. Goldstein, and Edward R.D. Scott. Main-group pallasites: Thermal history, relationship to IIIAB irons, and origin. Geochimica et Cosmochimica Acta, 74(15):4471-4492, aug 2010. ISSN 00167037. doi: 10.1016/j.gca.2010.04.016. URL http://linkinghub.elsevier.com/retrieve/pii/S0016703710002012. 